\title{
What Characterizes a Shadow Boundary under the Sun and Sky?
}

\author{
Xiang Huang ${ }^{b *}, \quad$ Gang Hua ${ }^{\natural}, \quad$ Jack Tumblin ${ }^{b}, \quad$ Lance Williams ${ }^{\sharp}$ \\ ${ }^{b}$ EECS Dept. \\ Northwestern University \\ ${ }^{\natural}$ Computer Science Dept. \\ ${ }^{\sharp}$ Multimedia Lab \\ Stevens Institute of Technology \\ Nokia Research Center \\ \{xhuang, jet\}@cs.northwestern.edu \\ ganghua@gmail.com \\ lance.williams@nokia.com
}

\begin{abstract}
Despite decades of study, robust shadow detection remains difficult, especially within a single color image. We describe a new approach to detect shadow boundaries in images of outdoor scenes lit only by the sun and sky. The method first extracts visual features of candidate edges that are motivated by physical models of illumination and occluders. We feed these features into a Support Vector Machine (SVM) that was trained to discriminate between mostlikely shadow-edge candidates and less-likely ones. Finally, we connect edges to help reject non-shadow edge candidates, and to encourage closed, connected shadow boundaries. On benchmark shadow-edge data sets from Lalonde et al. and Zhu et al., our method showed substantial improvements when compared to other recent shadowdetection methods based on statistical learning.
\end{abstract}

\section{Introduction}

In our visual world, shadows are ubiquitous, induced by directional lighting and occlusions that block its arrival at some of the scene's surfaces. Shadows offer important visual cues for human perception of shape, displacement, depth, and subtleties of contact between stacked objects, but the process of deriving these cues remains elusive.

Shadow detection has a wide variety of real-world applications including image relighting $[16,5]$, seamlessly combining existing photos and computer graphics [15], or detecting deceptive combinations of photos and graphics by image forensics and forgery detection [6], and video surveillance, where shadows that change with weather and time-of-day interfere with robust background subtraction [19]. Despite significant progress in shadow modeling in recent years $[20,12,24]$, shadow detection within a single image is inherently ill-posed, difficult, and worthy of exploration. Mimicking human-like methods may eventually

\footnotetext{
*Part of this work was carried out when $\mathrm{XH}$ was an intern at Nokia Research supervised by GH and LW.
}

provide human-like accuracy in this challenging task [13].

Due to the heterogenous lighting sources commonly found in real-world images, building a single shadow model that works perfectly for every individual photo is certainly difficult, and may be impossible. Instead, this paper addresses shadow boundaries in outdoor scenes with shadows caused by blocked sunlight. Since many consumer photographs capture travel and outdoor scenes, we believe studying outdoor sun-sky shadow detection has wide applications, e.g., vehicle and traffic sign recognition.

We begin with a careful examination of the shadows created outdoors by this two-source sun-and-sky model. From the physical model, we derive and design a set of discriminative and principled visual features to characterize outdoor shadow boundaries in photos. For example, the umbra (the inner, darker part of the shadow) receives only sky illumination, and tends to be darker and more bluish than the same surface strongly lit by the sun. We also mathematically model both the penumbra size (soft-shadow transition size) and the penumbra illumination intensity as part of our list of visual features.

We concatenate those physically modeled visual features to form a feature vector for each shadow boundary candidate. Next, we feed the feature vectors from a set of labeled training pixels of both shadow and non-shadow types into a Support Vector Machine (SVM) [3] to build an optimal discriminative model to differentiate shadow boundaries from non-shadow ones. As the visual features we chose offer high discriminative power, we believe that other machine learning algorithms might also work well for this shadow characterization, such as the AdaBoost [4] classifier we tested in the paper.

Next, we leverage our designed shadow boundary features and trained SVM model for shadow boundary detection. To build an efficient and effective shadow boundary detection for outdoor scenes, we employ both a prefiltering step and a post-linking step. Since shadow boundaries form visually apparent edges between differently illuminated image regions, our method begins by performing Canny edge detection [2] to filter out non-edge points that 
could never qualify as shadow boundaries. Next, it extracts from these edge points all visual features we inferred from physical models of shadow boundaries, and assembles them into feature vectors. Our previously-trained SVM model then scores each of these feature vectors to select candidate shadow boundary pixels.

Finally, the method applies both a high- and a lowconfidence threshold to the candidate boundary pixels to form maps of both the strong shadow edges and the weak shadow edges. The method then links together map elements to form continuous boundaries in a manner similar to the edge linking process of the Canny edge detector.

Our key contributions are:

- Careful models of outdoor illumination and geometry to produce reliably discriminative visual features to characterize shadow boundaries,

- Shadow detection with statistical learning methods based on these features, and

- Favorable results when compared to previouslypublished machine-learning approaches on two benchmark shadow-edge data sets from Zhu et al. [26] and Lalonde et al. [14].

Our proposed shadow boundary detection algorithm is a novel approach to combine statistical learning methods with visual features derived directly from physical models.

\section{Related Work}

Detection of moving shadows from multiple images attracted a substantial body of previous work. Notable papers include Weiss et al. [23], who proposed a method to remove shadow from multiple images with illumination changes by applying a median filter to the gradients of log-intensity image sequence. Sunkavalli et al. [21] gathered time-lapse video for days or weeks from a fixed outdoor camera, and then used PCA on the entire data set to separate reflectance values, shadows, and illumination over time for each pixel. Prati et al. [19] performed a survey and a good comparative study on different methods for shadow detection in videos for traffic flow analysis. Huerta et al. [11] devised a method for moving umbra shadow detection and removal by leveraging invariant color and gradient models, as well as temporal consistency of textures and gradients.

Yet shadow detection from a single image remains a very challenging problem. Most previous works compute 'shadow-free' images from color images, by finding $1 D$ or $2 D$ illumination-invariant images. For example, Finlayson et al. [7, 9, 8] proposed a method to compute 'shadow-free' images from an illumination-invariant grayscale image. But their assumptions of Planckian light sources and narrow-band camera sensors may not

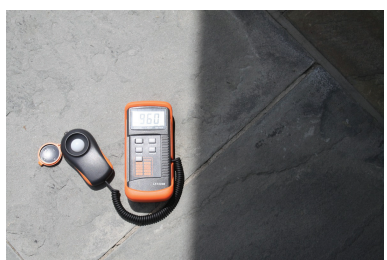

(a) Direct Sunlight: 96,000 lux (b) Within Shadow 14,400 lux

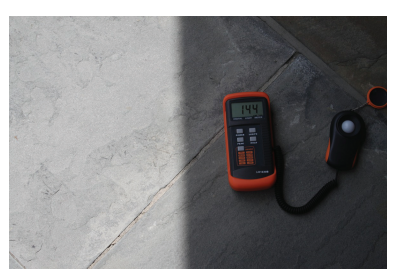

Figure 1. Illumination under sun and shadow at mid-day

be easily satisfiable for broader classes of single-image tasks. Maxwell et al. [18] described a bi-illuminant dichromatic reflection model to project an RGB image to an illumination-invariant image, but only with $2 D$ color channels. Tian et al. [22] preprocessed an input RGB image to illumination-invariant grayscale image, then segmented the grayscale image to sub-regions, and detected shadows by tricolor attenuation modeled by spectral power of sunlight and skylight. However, the initial illumination-invariant image may not be reliable, and the tricolor attenuation model may be inaccurate in sunrise and sunset when spectral differences grow.

Other researchers also leveraged statistical machine learning for natural image boundary detection, such as work by Martin et al. [17], and also attempted shadow detection from single images, either for aerial photographs [25], color images [14] or monochromatic images [26]. Although each demonstrated promising performance, the visual features they adopted for training the classifiers relied on design intuitions rather than the physics of light transport through the scene. It remains a question how to design discriminative features for shadow detection based on real-world physical models.

\section{Physical model of outdoor shadows}

In this section, we examine the physical properties of outdoor shadows under the sun and sky. Perhaps the most obvious difference between the sun and sky illumination is the intensity change. For example, the Sinometer LX1330B light meter shown in Fig. 1 measured incident illumination of 96,000 lux in direct sunlight outdoors at mid-day, but when placed in the outdoor shadow of a large building, illumination fell to just 14, 400 lux.

We also measure illumination changes between outdoor sunlight and shadowed regions using a Canon EOS Rebel T1i DSLR camera, as shown in Fig. 2(a). This outdoor photo captures a large-building shadow cast on a flat piece of white letter size paper, arranged nearly perpendicular to the camera to ensure that image intensity changes could be attributed solely to the illumination change from sunlight to shadow.

Examining the progressive change of radiance values along the scan line $x$ perpendicular to the shadow bound- 


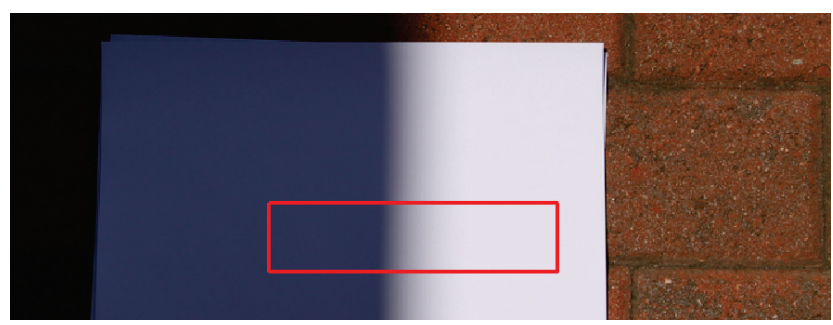

(a) Shadow cast on a piece of white paper by building roof

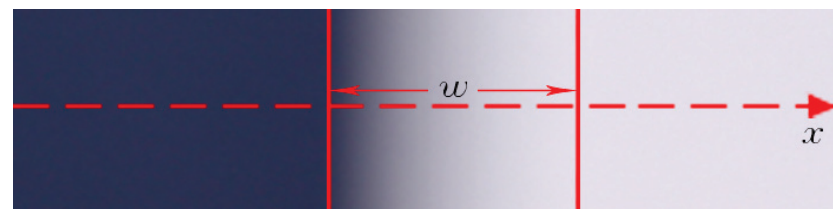

(b) Crop a rectangular region that includes the shadow boundary

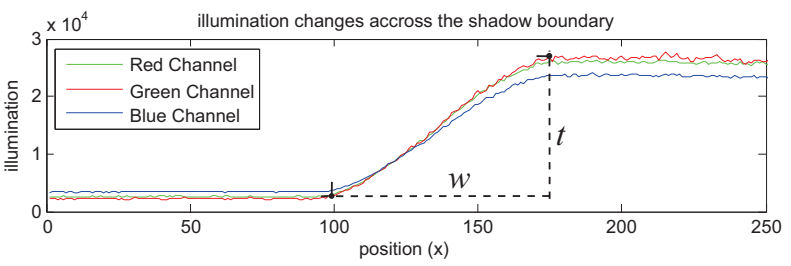

(c) Shadow-to-sunlight illumination changes measured along the scan-line

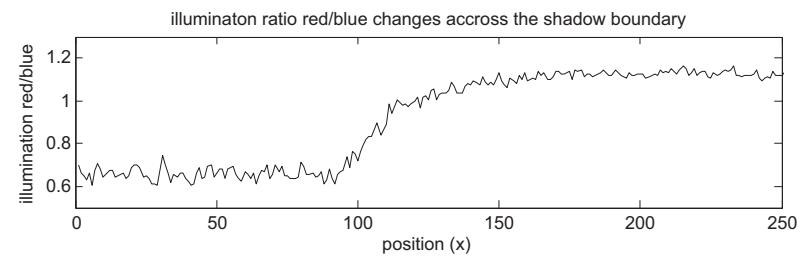

(d) Scan-line red/blue ratio: 0.65 in sunlight, 1.1 in shadow

Figure 2. Illumination change across the shadow boundary

ary in Fig. 2(b) reveals important color differences between sunlight and shadow. We took this photo in raw format with the T1i camera, and then convert it to a 'linearized' 16-bit TIFF image whose pixel values are linearly proportional to the scene radiance. We further calibrate the color of the paper by comparing the average pixel value of a patch of paper with that of a gray card under the same illumination. We scale each color channel of the image according to the paper color to obtain an illumination-only image, and thus ensure that pixel RGB values in Fig. 2(c) are directly proportional to illumination at each point on the paper.

Careful examination of RGB scanline values plotted in Fig. 2(c) confirms shadow illumination has a blue cast, matching the sky's blue color caused by Rayleigh scattering $[10,1]$. By comparison, the sun-and-sky lit portion shows increased strength in red/yellow components, greatly increasing the ratio of red/blue at each pixel, plotted in Fig.2(d). For the mid-range color balance we chose, the red/blue ratio grows from approximately 0.65 in the shadowed region lit only by sky upwards to approximately 1.1

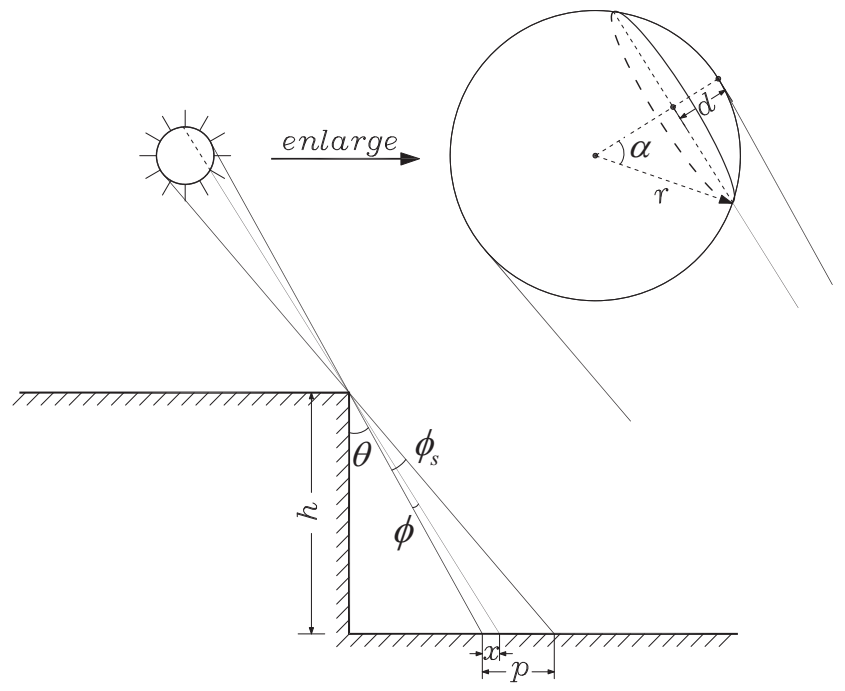

Figure 3. Penumbra size and illumination intensity

in the region lit by direct sunlight and sky combined.

Shadows also vary with the shape and distance to the occluder that causes them. For example, outdoors on a sunny day, you may notice that shadows cast by low buildings have much sharper shadow boundaries than shadows cast by tall buildings at the same time of day. At noon, building shadows reach their sharpest, shortest shape, but as the day stretches towards dusk, shadows lengthen and get less distinct as their penumbrae get wider and illumination intensity falls. Simple geometry links the shadow sharpness, which we will call penumbra width $p$, the occluder height $h$, and the sunlight's incidence angle $\theta$, in our Theorem 1:

Theorem 1. The shadow sharpness $p$ is linearly proportional to the occluder height $h$ and inversely proportional to the square of the cosine of the sun's incidence angle $\theta$.

Proof. In the scene depicted in Fig. 3 a building of height $h$ occludes the sun and casts a shadow on the ground with penumbra size $p$. Given the sun's angular size $\phi_{s} \approx 0.0093$ radians ( 0.53 degrees), when the incidence angle $\theta$ between a sun ray and the normal of the ground increases by a small amount $\phi_{s}$, a point $x$ moves from the $x=0$ (umbra border) to $x=p$ (sunlit border). The penumbra size or 'shadow sharpness' is:

$$
\begin{aligned}
p & =h \tan \left(\theta+\phi_{s}\right)-h \tan \theta \\
& \approx h \phi_{s} / \cos ^{2} \theta .
\end{aligned}
$$

The approximation holds as $\tan \theta$ has derivative $1 / \cos ^{2} \theta$ and the sun's angular extent $\phi_{s}$ spans only 0.0093 radians.

The theorem was written for a building on level ground, but also holds for general occluders (i.e., ground, utility pole, car) and shadows cast on other surfaces such as walls, 
by defining $h$ as the distance between the occluder and shadowed surface, and $\theta$ as the incidence angle between the sun ray and the surface normal of the shadowed surface.

Together, Theorem 1 and Equation (1) explain why lower buildings have sharper shadows than tall ones, as shadow sharpness $p$ is directly proportional to building height $h$.

We could also infer from Theorem 1 that the shadow sharpness $p$ is constant for a building with same height, or vary smoothly along the shadow boundary, because any physically realizable occluder such as a building must have a continuous height $h$. Consequently, the shadow transition width $w$ (in pixels) in an image is smooth at locally flat surfaces in the scene, such as wall and floor. Furthermore, the average change rate of the pixel values on a scanline crossing this shadow boundary, shown as $t / w$ in the scanline plot of Fig. 2(c), must be continuous, and must be inversely proportional to shadow sharpness $w$ because $t$ is constant everywhere in the scene (we assume all shadows receive the same sky-only illumination).

Later, these two continuous properties simplify the edge linking process, and will resolve any ambiguities at T-junctions between shadow boundaries and reflectance edges, as demonstrated in our experiments in Section 6.

Fig. 2(c) shows a slightly curved and symmetric intensity profile for the penumbra, with maximal slope at the middle of the penumbra. Theorem 2 below describes the intensity change rate in the penumbra.

Theorem 2. Within any image of a scene lit only by the sun and sky, if we choose any straight line perpendicular to a shadow boundary, then the illumination intensity change (or radiance) has a rate proportional to sqrt $(1-(2 x / p-$ $\left.1)^{2}\right)$ along that line through the penumbra, where $x$ is the distance to the umbra, and $p$ is the penumbra size.

Proof. As shown in Fig. 3, when the position $x$ along the scan line (orthogonal to the shadow boundary) changes from 0 to $p$, the visible portion of the sun's surface that an observer can see from point $x$ will change as well. At $x=0$, we cannot see the sun at all. At $x=p$, we see half of the sun's surface with area $2 \pi r^{2}$ for sun radius $r$. At $0<x<p$ within the penumbra, we can see only a portion of the sun's surface: a half-spherical cap with height $d$ as shown in Fig. 3. According to the triangle similarity, we have $d /(2 r)=\phi / \phi_{s}=x / p$, thus the visible half spherical cap has area $\pi r d=2 \pi r^{2} x / p$ for $0<x<p$.

When we move from $x$ to $x+\Delta x$ for a small amount $\Delta x$, we will see an increased sun area of $2 \pi r^{2} \Delta x / p$, the surface area of which has angle $(\pi / 2-\alpha)$ to the incident sun ray. The increased visible surface will add an illumination intensity amount that is proportional to $\left(2 \pi r^{2} \Delta x / p\right) * \cos (\pi / 2-$ $\alpha$ ). From Fig. 3, we can see $\cos \alpha=1-d / r=1-2 x / p$, thus the illumination intensity change rate with respect to $x$ is proportional to $\cos (\pi / 2-\alpha)=\operatorname{sqrt}\left(1-(2 x / p-1)^{2}\right)$. Note the illumination intensity within the penumbra has its largest slope at the penumbra's centerline $x=p / 2$.

Theorem 2 demonstrates that the illumination measured across the penumbrae for any shadow has the same fixed shape, which may be another clue to distinguish shadow boundaries from reflectance boundaries, without regard to the orientation, shape, or placement of either kind of edge. Of course, the shape of the shadow boundary may be smoothed by blur from a poorly focussed camera, and its intensity profile may get distorted by camera vignetting or camera sensors with nonlinear responses to radiance, but underneath these distortions, shadows on flat surfaces will still exhibit the characteristic features described by Theorem 2 .

These physical properties of outdoor shadows motivated and led us to design a set of discriminative visual features to characterize shadow boundaries in forms suitable for use with machine-learning methods.

\section{Discriminative Shadow Boundary Features}

Very few pixels in an image describe an edge or a boundary of any kind. Our method begins by applying the Canny edge detection algorithm to identify all the pixels that might be part of an edge, without regard to the cause of those edges. In this section we exploit our observations about shadows from the last section to define several visual features. Each one offers indicators about the pixel's suitability as a shadow-edge, and we will use sets of these features to train our chosen machine learning method (SVM) to distinguish between edge-candidate pixels that are likely or unlikely to be part of a shadow edge.

The first visual feature for candidate-edge pixels measure the illumination ratio of the sun and sky, which differ substantially across a shadow boundary. We compute the Gaussian weighted average of pixels on both sides of the edge: the bright, sunlit side has weighted average $H_{r}, H_{g}, H_{b}$ for red, green and blue channels respectively, and the dark, shadowed side has weighted average $L_{r}, L_{g}, L_{b}$. We then compute the intensity ratio $\left(t_{r}, t_{g}, t_{b}\right)=\left(L_{r} / H_{r}, L_{g} / H_{g}, L_{b} / H_{b}\right)$. Assuming the reflectance is locally uniform near the shadow boundary, the intensity ratio will match the illumination ratio of the sun and sky when measured across the shadow boundary. Thus we can reasonably expect $t_{r}>t_{g}>t_{b}$ at shadow boundaries, as the shadow, lit only by the sky, is more bluish than the sunlit side. Finally, we compute the feature vector as $\left(t, t_{r b}, t_{g b}\right)=\left(\left(t_{r}+t_{g}+t_{b}\right) / 3, t_{r} / t_{b}, t_{g} / t_{b}\right)$. This feature vector encodes the sun and sky illumination difference and hence offers a more easily-discriminated cue than simply using $\left(t_{r}, t_{g}, t_{b}\right)$ directly. 


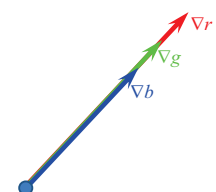

(a)

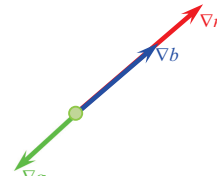

(b)

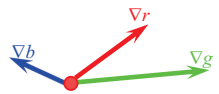

(c)
Figure 4. Image gradients computed separately for each RGB color channel. (a)Shadow edge: same direction. (b)Possible other edges: opposite but collinear direction (e.g., gradient in green channel is opposite to gradient in blue channel at the boundary between pure green material and pure red material). (c)Possible other edges: more general cases with non-collinear directions.

The second visual feature compares the gradient of illumination of the sun to help identify shadow boundaries. First, we compute the color gradient magnitude $\left(s_{r}, s_{g}, s_{b}\right)$ at each candidate edge pixel. As shown in Fig. 2(c), $\left(s_{r}, s_{g}, s_{b}\right)$ corresponds to the gradient of the sun's illumination in the penumbra, but includes scaling by the local reflectance values. We defined this feature vector as $\left(\delta_{r}, \delta_{g}, \delta_{b}\right)=\left(s_{r} / H_{r}, s_{g} / H_{g}, s_{b} / H_{b}\right)$ to cancel the reflectance, and to leave behind only the gradient of illumination.

We construct our third set of features from the color gradient direction $\left(\gamma_{r}, \gamma_{g}, \gamma_{b}\right)$. For any candidate edge pixel at a shadow boundary where reflectance is locally constant, the image gradient should have the same direction in all color channels, as the RGB illumination gradients are all perpendicular to the shadow boundary. Other kinds of edges may lack this property, as demonstrated in Fig. 4. The third feature vector exploits this distinction by measuring the gradient's directional difference for each color as $\left(\gamma_{r g}, \gamma_{g b}, \gamma_{b r}\right)$, where $\gamma_{r g}=\min \left(\left|\gamma_{r}-\gamma_{g}\right|, 2 \pi-\left|\gamma_{r}-\gamma_{g}\right|\right)$. We found that feeding the SVM with these angular differences $\left(\gamma_{r g}, \gamma_{g b}, \gamma_{b r}\right)$ gave better results than supplying the original angles $\left(\gamma_{r}, \gamma_{g}, \gamma_{b}\right)$ alone, as the the former are rotationally invariant. In our experiment, the variance of gradient direction in different color channels for non-shadow edges is on average around 15 times larger than that of shadow edges.

We derive the fourth visual feature vector from the edge width values in each color channel $\left(w_{r}, w_{g}, w_{b}\right)$. As shown in Fig. 2(c), a shadow boundary should have the same width for all color channels. We compute this fourth feature vector as $\left(w, w_{r g}, w_{r b}\right)$, where $w=\left(w_{r}+w_{g}+w_{b}\right) / 3, w_{r g}=$ $\left|w_{r} / w_{g}\right|, w_{r b}=\left|w_{r} / w_{b}\right|$. Note Theorem 1 also indicates that shadow width is a continuous parameter thus we could also use the edge parameter for shadow edge linking.

In summary, we build a visual feature vector $\left(t, t_{r b}, t_{g b}, \delta_{r}, \delta_{g}, \delta_{b}, \gamma_{r g}, \gamma_{g b}, \gamma_{b r}, w, w_{r g}, w_{r b}\right)$ at three scales at each edge pixel. If we choose an edge pixel actually located at a shadow boundary, then its $36 D(12 D$ times three scales) visual-feature vector will contain shadow-

\begin{tabular}{|c|c|c|c|c|}
\hline \multirow{2}{*}{ Dataset } & \multicolumn{2}{|c|}{ AdaBoost } & \multicolumn{2}{c|}{ SVM } \\
\cline { 2 - 5 } & Ours & Lalonde's & Ours & Lalonde's \\
\hline LAL & $78.75 \%$ & $74.06 \%$ & $81.63 \%$ & $77.56 \%$ \\
ZHU & $83.83 \%$ & $79.50 \%$ & $85.92 \%$ & $81.52 \%$ \\
\hline
\end{tabular}

Table 1. Shadow classification accuracy at equal error rate (EER) comparing with [14] in dataset LAL [14] and dataset ZHU [26].

edge-like values for its ratio between the sun illumination and sky illumination, the gradient of the sun illumination, the color gradient direction, and the shadow sharpness information. These features, each one motivated from the physical model and phenomena described in the previous section will offer their own distinctive characterizations of shadow boundaries.

\section{Shadow Boundary Detection System}

Given a labeled training dataset, such as those from Zhu et al. [26] and Lalonde et al. [14], we can train an SVM classifier for shadow boundary detection using the $36 \mathrm{D}$ features extracted from labeled shadow and non-shadow Canny edges. We adopted the LIBSVM toolkit [3] to train the SVM model with probabilistic output, utilizing a Gaussian $\mathrm{RBF}$ kernel. Note our features can work with any other type of classifier such as the AdaBoost; we adopted SVM due to its better accuracy manifested in our experiments.

We detect the shadows in a test image with the following procedures. We first pre-filter the input image using the Canny edge detector to select all the edge-candidate pixels in the image. We will select all our initial shadow-edge pixels from this set. Then we compute a $36 D$ feature vector for each of these edge-candidate pixels, and feed the feature vectors to the pre-trained SVM classifier. For each edge pixel, we extract a probability from the SVM output for the likelihood that the edge pixel is part of a shadow boundary. We then connect together highly-likely edges with an edge linking similar to the one employed by Canny. Each linked edge begins at a pixel that the SVM identified as a highly-probable shadow-edge member. We link this to nearby pixels with middle-range shadow-edge probabilities that extend the shadow edge most consistently. Note the high probability and low probability threshold could also be learned from the training set, as discussed in more detail with the experiments in Section 6.

Next, we present extensive experiments to demonstrate the efficacy of the shadow features we designed and the high performance of our shadow detection system, with comparison to previously published methods.

\section{Experiments, results, and discussion}

To validate the efficacy of the proposed $36 D$ features, we compare them with the $48 D$ features proposed by 


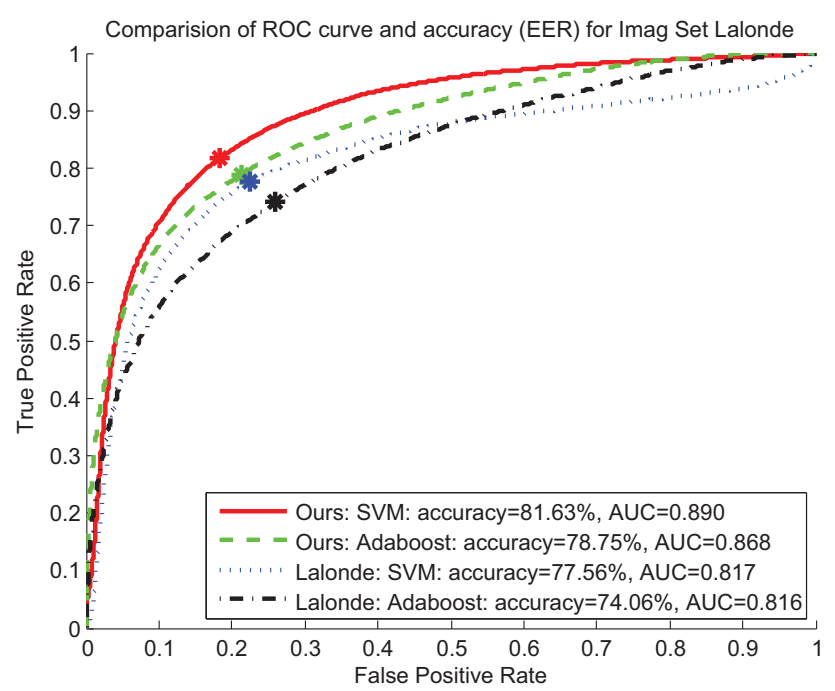

Figure 5. ROC curves on dataset Lalonde.

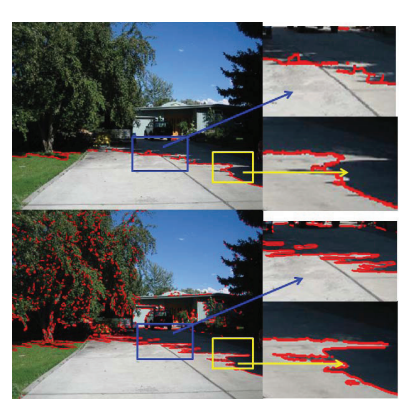

(a)

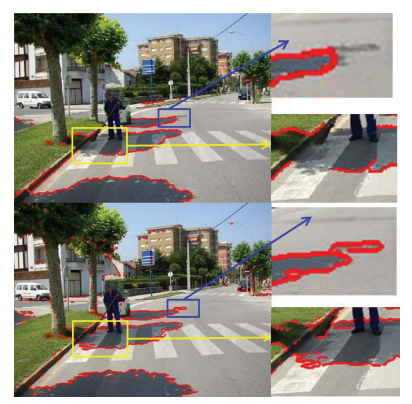

(b)
Figure 7. Shadow detection results compared with Lalonde et al. [14]. Lalonde et al.'s result is shown on top, ours on bottom. The shadow boundaries are labeled with red lines.

Lalonde et al. [14]. The comparison is performed on two datasets from Lalonde et al. [14], and Zhu et al. [26]. We call them dataset $L A L$ and dataset $Z H U$, respectively.

Dataset LAL consists of 135 images in total. We randomly selected 100 images for training and the other 35 for testing. Dataset ZHU contains 162 images. Again, we randomly selected 100 images from it for training and the remaining 62 for testing. In both of the experiments, we randomly sampled 30,000 shadow edges and 30, 000 nonshadow edges from the 100 images for training. For testing, we randomly sampled 10, 000 shadow and 10, 000 nonshadow edges from the 35 test images in dataset LAL, and 20, 000 shadow and 20,000 non-shadow edges from the 62 test images in dataset ZHU.

Since Lalonde et al. [14] used an AdaBoost classifier to train their shadow boundary detector, in our experiments, we also performed experiments with the same version of the AdaBoost classifier. For training the SVM classifier, we leverage LIBSVM to train an SVM classifier with proba-

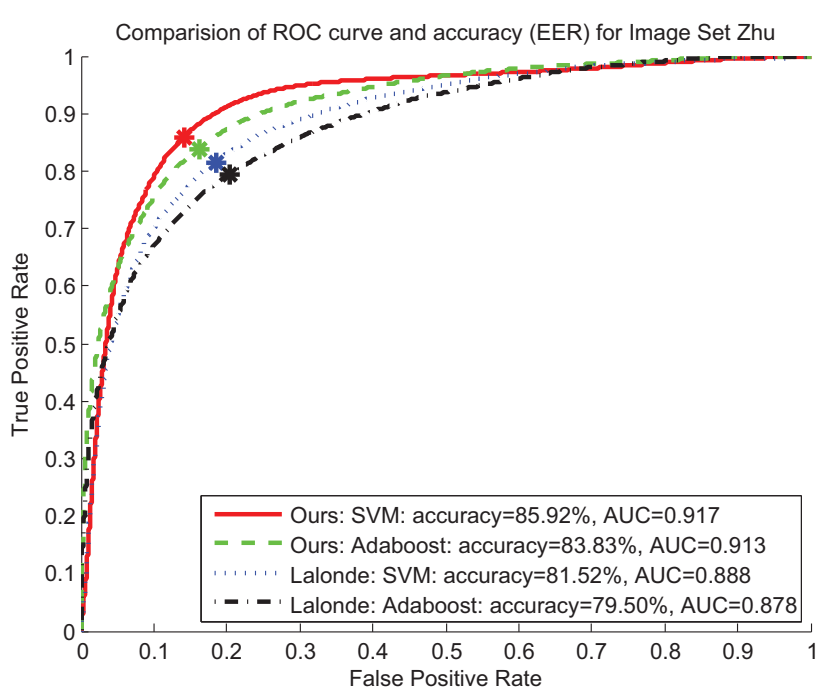

Figure 6. ROC curves on dataset Zhu.

bility output and RBF kernel from the training data, using our features and Lalonde et al.'s features, respectively. Note no matter what classifier we adopted, we could still run our edge linking sub-system to further improve the results.

\subsection{Quantitative Results}

Fig. 5 and Fig. 6 present the ROC curves and the AUC (Area Under Curve) from the output of the trained classifiers on dataset LAL and ZHU, respectively. Note we do not perform any linking on the shadow edges for this set of experiments to better evaluate the raw features. Each figure presents four ROC curves, which correspond to the combination of two classifiers (SVM and AdaBoost), and two feature vectors (ours and Lalonde et al.'s). The curves clearly demonstrate that the classifiers trained using our $36 D$ features always outperform their competing classifiers using Lalonde et al.'s $48 D$ feature by a margin $>4.0 \%$ in absolute value. We also observe that using the same type of features, the SVM always induced higher accuracy than AdaBoost. As a matter of fact, the SVM Classifiers with Lalonde et al.'s features also achieved inferior results than the AdaBoost classifiers trained using our $36 D$ feature vector. These observations are consistent on the two datasets. We further summarize the shadow classification accuracy at equal error rate (ERR) in Table 1. All these results present very strong evidence that our proposed $36 D$ features are both more discriminative and more compact than the $48 \mathrm{D}$ features proposed by Lalonde et al. [14] for shadow detection.

\subsection{Qualitative Results}

We qualitatively compare the results of our full shadow detection system in Fig. 7 with Lalonde et al.'s system [14]. We can observe that our algorithm performs well for ground 
shadows and yet detects non-ground shadows well when compared to Lalonde et al. Notice that our shadow detection is more accurate in terms of localization, especially in those blue rectangular regions. We can also observe that we missed fewer shadow boundaries.

We also attack a challenging case pointed out by Zhu et al. [26], where the shadows are cast on water, as shown in Fig. 8. While Zhu et al.'s features do not capture the properties of shadows in the water, our feature vector captures it well, since the shadow is still more bluish than the sunlit area. We further show in Fig. 9 our shadow detection works well for many challenging cases. Our approach successfully detects shadows in different contexts, including foliage shadows on concrete and tarmac, in close-ups and aerial photos. This shows our features are robust in a wide variety of outdoor environments.

\subsection{Discussions}

Our feature vector may not work well for indoor photographs as shown in Fig. 10. That is quite understandable because the sun and sky model can no longer characterize the shadows. Our feature vector may also fail for very faint shadows, where the sky is not totally occluded by very small occluders, such as a tree leaf.

Our physically based shadow boundary model may be further explored for scene understanding. For example, from Theorem 1, we could compare the ground shadow sharpness of two buildings to reveal their relative heights, and use that information to assist $3 D$ reconstruction.

Our model may also be used for better shadow removal. Most shadow removal techniques smooth out the gradient at the shadow boundary, and re-integrate the modified gradient to form a shadow-free image. The reflectance gradient will be lost and the shadow boundary will be blurred. A better approach would fit the illumination gradient according to Theorem 2 and remove shadows accordingly.

\section{Conclusion and future work}

We carefully examined the physical properties and models of outdoor shadows lit only by the sun and sky. These studies reveal a set of discriminative features for shadow detection. Based on them, we developed an end-to-end shadow boundary system with three steps, i.e., pre-filtering, shadow boundary classification, and post-linking. Our algorithm compares favorably with the state-of-the-art benchmark shadow data-sets. Future work will explore the use of filter banks to match the shape described by Theorem 2 to increase both the robustness and localization accuracy of our shadow detection system. We will also further explore machine learning based shadow edge linking algorithms in our future work.

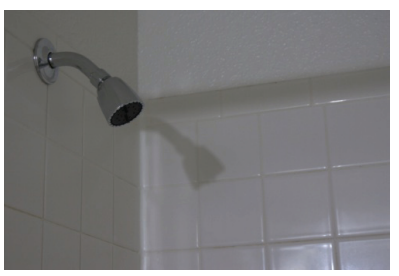

(a)

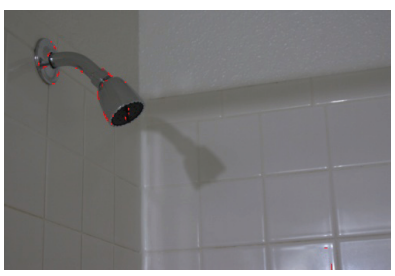

(b)
Figure 10. Failure examples

\section{Acknowledgement}

We thank Zhiyao Duan and Dr. Thommen Korah for helpful discussions, and Wei Zhou for help with Fig. 3. This project was supported in part by Nokia and by NSF grant number 0535236 .

\section{References}

[1] http://hyperphysics.phy-astr.gsu.edu/ hbase/atmos/blusky.html.

[2] J. Canny. A Computational Approach to Edge Detection. PAMI, PAMI-8(6):679-698, Nov. 1986.

[3] Chih-Chung Chang and C.-J. Lin. $\{$ LIBSVM $\}$ : a library for support vector machines. 2001.

[4] M. Collins, R. E. Schapire, and Y. Singer. Logistic Regression , AdaBoost and Bregman Distances. Machine Learning, pages 253-285, 2002.

[5] P. Debevec. Virtual Cinematography: Relighting through Computation. Computer, 39(8):57-65, Aug. 2006.

[6] H. Farid. Digital doctoring: how to tell the real from the fake. Significance, 3(4):162-166, Dec. 2006.

[7] G. Finlayson, S. Hordley, and M. Drew. Removing shadows from images. ECCV, pages 129-132, 2002.

[8] G. D. Finlayson, M. S. Drew, and C. Lu. Entropy Minimization for Shadow Removal. IJCV, 85(1):35-57, May 2009.

[9] G. D. Finlayson, S. D. Hordley, C. Lu, and M. S. Drew. On the removal of shadows from images. PAMI, pages 1-11, 2006.

[10] B. R. Holstein. Blue skies and effective interactions. American Journal of Physics, 67(5):422, May 1999.

[11] I. Huerta, M. Holte, T. Moeslund, and J. Gonzalez. Detection and removal of chromatic moving shadows in surveillance scenarios. In ICCV, Tokyo, Japan, October 2009.

[12] T. Kim and K.-S. Hong. A practical single image based approach for estimating illumination distribution from shadows. ICCV, 2005.

[13] F. A. A. Kingdom, C. Beauce, and L. Hunter. Colour vision brings clarity to shadows. Perception, 33(8):907-914, 2004.

[14] J.-F. Lalonde, A. A. Efros, and S. G. Narasimhan. Detecting ground shadows in outdoor consumer photographs. In ECCV, pages 1-14, 2010.

[15] C. Loscos, M.-C. Frasson, G. Drettakis, B. Walter, $\mathrm{X}$. Granier, and P. Poulin. Interactive virtual relighting and remodeling of real scenes. EGSR, 10:235-246, Jun 1999. 


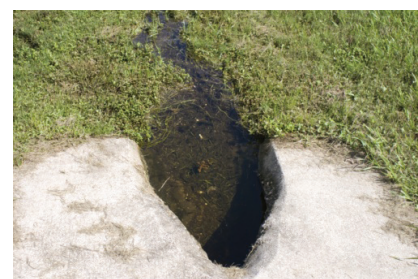

(a) Original

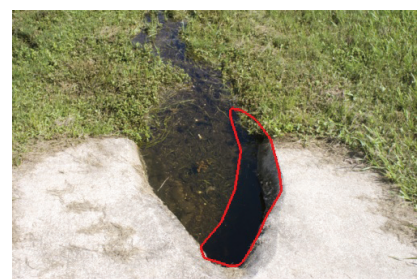

(b) Ground Truth

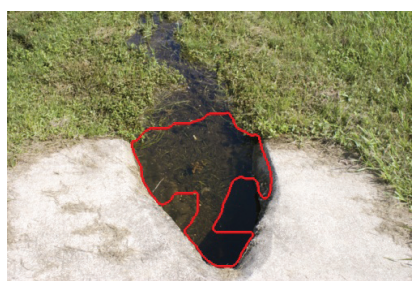

(c) Zhu et al.'s result

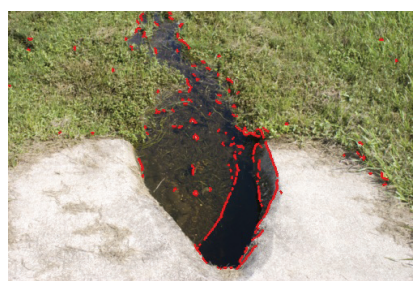

(d) Our result

Figure 8. Shadow detection results comparing with Zhu et al. [26]
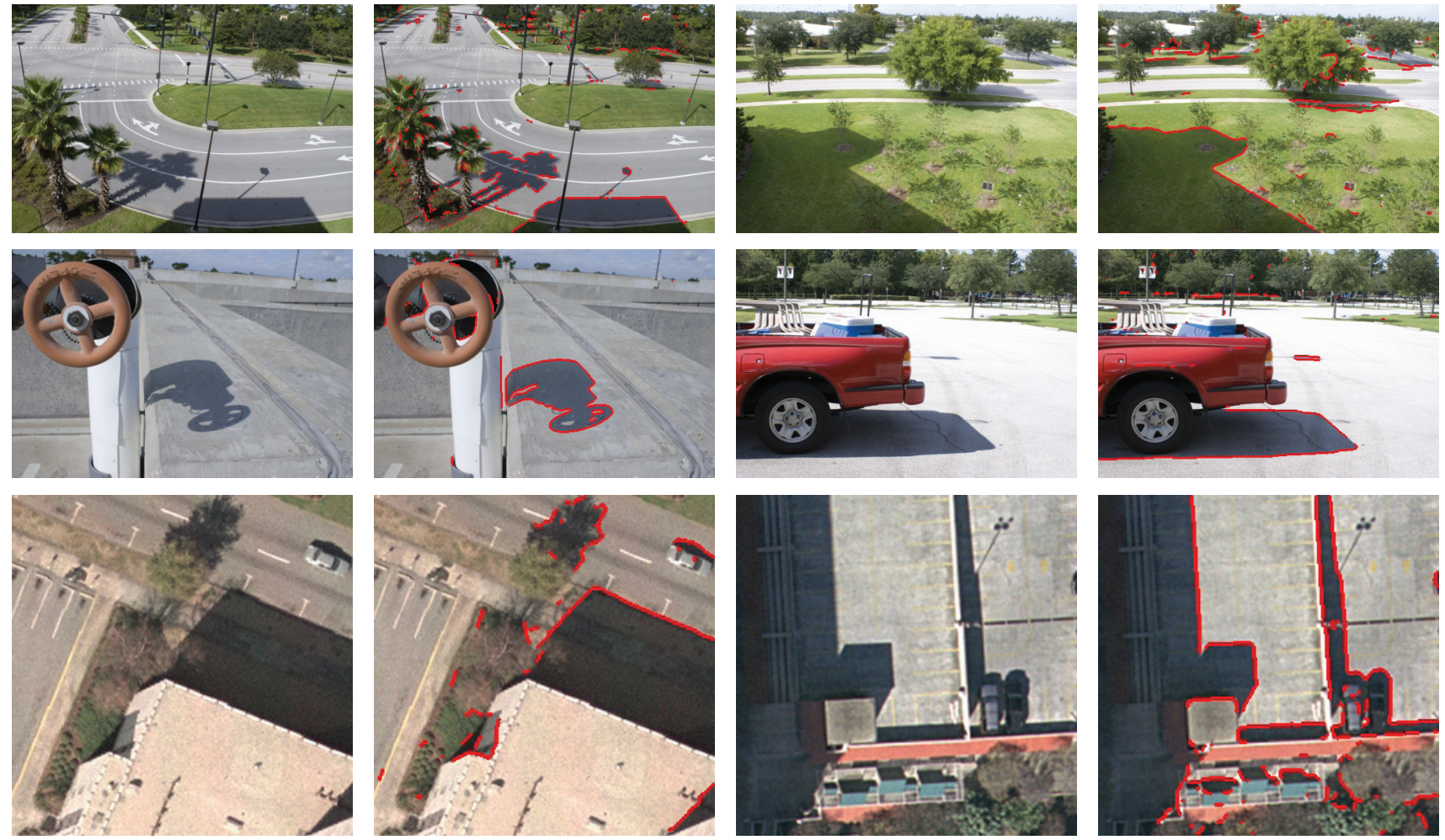

Figure 9. More shadow detection results on images from [26] and [14]. First and third columns are input images. Second and fourth columns are detected shadows.

[16] C. Madsen and Rune Laursen. Image relighting: Getting the sun to set in an image taken at noon. Conference on Pattern Recognition and Image, 2004.

[17] D. R. Martin, C. C. Fowlkes, and J. Malik. Learning to detect natural image boundaries using local brightness, color, and texture cues. PAMI, 26(5):530-49, May 2004.

[18] B. A. Maxwell, R. M. Friedhoff, and C. A. Smith. A biilluminant dichromatic reflection model for understanding images. In $C V P R$, pages 1-8. IEEE, June 2008.

[19] A. Prati, I. Mikic, C. Grana, and M. Trivedi. Shadow detection algorithms for traffic flow analysis: a comparative study. In Proc. IEEE Intelligent Transportation Systems Conf, pages 340-345, 2001.

[20] I. Sato, Y. Sato, and K. Ikeuchi. Illumination from shadows. PAMI, 57(1):215-300, Mar. 2003.
[21] K. Sunkavalli, W. Matusik, H. Pfister, and S. Rusinkiewicz. Factored time-lapse video. SIGGRAPH, 26(3), Aug. 2007.

[22] J. Tian, J. Sun, and Y. Tang. Tricolor Attenuation Model for Shadow Detection. IEEE Tran. on Image Processing, 18(10):2355-2363, 2009.

[23] Y. Weiss. Deriving intrinsic images from image sequences. In $I C C V$, pages 68-75. IEEE, 2001.

[24] Y. Yamashita and F. Sakaue. Recovering 3D Shape and Light Source Positions from Non-Planar Shadows. ICPR, pages $3-$ 6, 2010.

[25] J. Yao and Z. Zhang. Hierarchical shadow detection for color aerial images. CVIU, 102(1):60-69, Apr. 2006.

[26] J. Zhu, K. Samuel, S. Masood, and M. Tappen. Learning to Recognize Shadows in Monochromatic Natural Images. In CVPR, 2010. 